\title{
Vernier-cascade label-free biosensor with integrated arrayed waveguide grating for wavelength interrogation with low-cost broadband source
}

\author{
Tom Claes ${ }^{1,2, *}$, Wim Bogaerts ${ }^{1,2}$ and Peter Bienstman ${ }^{1,2}$ \\ ${ }^{1}$ Photonics Research Group, Department of Information Technology \\ Ghent University - IMEC, Sint-Pietersnieuwstraat 41, 9000 Gent, Belgium \\ ${ }^{2}$ Center for Nano- and Biophotonics (NB-Photonics), Ghent University \\ *Corresponding author: tom.claes@intec.ugent.be
}

Compiled July 25, 2011

\begin{abstract}
Recently, cheap silicon-on-insulator label-free biosensors have been demonstrated that allow fast and accurate quantitative detection of biologically relevant molecules for applications in medical diagnostics and drug development. However, whereas the sensor chip can be made cheap, an expensive tunable laser is typically required to accurately monitor spectral shifts in the sensor's transmission spectrum (wavelength interrogation). To address this issue, we integrated a very sensitive Vernier-cascade sensor with an arrayed waveguide grating spectral filter that divides the sensor's transmission spectrum in multiple wavelength channels and transmits them to spatially separated output ports, allowing wavelength interrogation with a much cheaper broadband light source. Experiments show that this sensor can monitor refractive index changes of watery solutions in real-time with a detection limit $\left(1.6 \cdot 10^{-5} R I U\right)$ competitive with more expensive interrogation schemes, indicating its applicability in low-cost label-free biosensing. The relaxation on the complexity of the source moreover offers the prospect to integrate source and detectors to further reduce the device cost and to increase its portability. (C) 2011 Optical Society of America

OCIS codes: $130.6010,130.7408,280.1415$.
\end{abstract}

More efficient methods to detect biologically relevant molecules in a fluid are increasingly demanded for applications in e.g. medical diagnostics and drug development. Especially photonic label-free biosensors receive a lot of attention for allowing fast, cheap and quantitative measurements in real-time [1]. They contain waveguides with immobilized receptor molecules and can efficiently convert refractive index changes caused by selective molecular binding to a measurable spectral shift in their optical transmission. Silicon-on-insulator (SOI) is a material system with many assets for this application, as its high index contrast allows the incorporation of many sensors on a chip to simultaneously measure multiple parameters. Moreover it permits cheap and reliable fabrication in high volume with CMOS-compatible processes. Recently, we and other research groups have demonstrated SOI sensor matrices capable of real-time multiparameter measurements with excellent detection limits $[2-4]$.

However, although the sensor chips can be made cheap, an expensive high-end tunable laser is typically required to repeatedly scan the transducers' transmission spectra with high resolution in order to accurately monitor their spectral shifts. One way to reduce the total cost of the device is to apply intensity interrogation instead of wavelength interrogation, by measuring the transducer's transmission with a fixed-wavelength laser [5] or broadband source [6,7], but this can be impractical due to the non-linear response and the need to align the transducer's transmission spectrum to the spectrum of the source or detector. This can be overcome by implementing wavelength interrogation with a cheap broadband source and on-chip wavelength filtering to divide the transducer's transmission spectrum in multiple wavelength channels and distribute them to spatially separated output ports. To retain a low detection limit, this concept requires a very sensitive sensor with a transmission spectrum that can be resolved with an on-chip spectral filter. In [8] a SOI ring resonator sensor with integrated photonic crystal filter is proposed, but the sharp resonances of that sensor put very stringent requirements on the filter and so far no low detection limits have been shown.

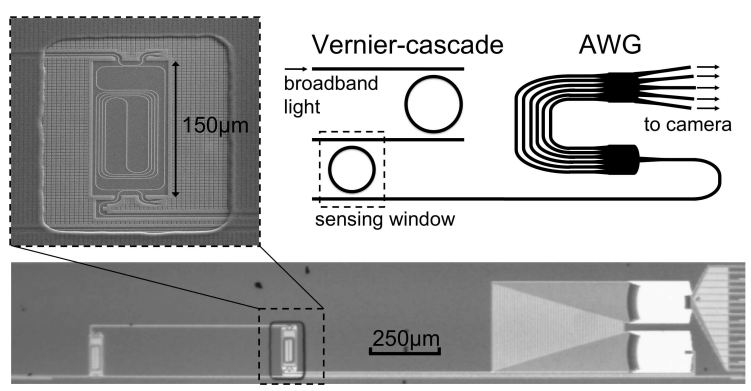

Fig. 1. The transmission spectrum of a Vernier-cascade sensor can be measured with a low-cost broadband light source by dividing it into different wavelength channels with an integrated arrayed waveguide grating (AWG) that distributes them to spatially separated output ports that can be monitored in parallel with a camera. The micrograph of the silicon-on-insulator chip shows the compact folded cavities of the ring resonators constituting the Vernier-cascade that is connected to a dense 16-channel AWG. The chip is covered with a polymer cladding except for a window to the second resonator to allow its evanescent field to interact with the fluid. 
We chose to integrate a Vernier-cascade sensor $[9,10]$ with a well-known and compact arrayed waveguide grating (AWG) [11], as depicted in Fig. 1. The Verniercascade can be made at least an order of magnitude more sensitive than a ring resonator sensor, with sensitivities as large as several thousands of nm/RIU. Moreover it can be designed to have a transmission spectrum with an easy-to-resolve periodic envelope signal superposed on sharp constituent peaks, as we showed in [9]. The sensor consists of two ring resonators with different roundtrips that are cascaded so that the drop signal of the first serves as the input of the second. The evanescent field of only one of the two resonators is allowed to interact with the refractive index of the fluid, of which a small change will cause a small shift of the resonance wavelengths of this resonator relative to that of the other, which will result in a much larger shift of the envelope signal of the Vernier-cascade. The output of the AWG's ports are monitored in parallel with an infrared camera, making this interrogation scheme compatible with the simultaneous read-out of multiple sensors on the same chip.

Our implementation of the device consists of $450 \mathrm{~nm}$ wide single-mode waveguides defined in the $220 \mathrm{~nm}$ high silicon top layer of a SOI wafer with $2 \mu \mathrm{m}$ buried oxide using CMOS-compatible $193 \mathrm{~nm}$ optical lithography and dry etching. A $600 \mathrm{~nm}$ thick cladding of a benzocyclobutene-based polymer with low water absorption covers the complete chip except for a window to the second resonator. The sensor was designed following previously published guidelines [9]. The filter and sensor resonator roundtrip lengths were chosen respectively $1271 \mu \mathrm{m}$ and $1257 \mu \mathrm{m}$ so that the sensor would have a clearly visible envelope signal and would have a large sensitivity, while retaining acceptable roundtrip losses. Both resonator cavities were folded with a $6 \mu \mathrm{m}$ bend radius to reduce their footprint to less than $7500 \mu \mathrm{m}^{2}$ (Fig. 1). The coupling of each resonator to its two access waveguides is matched to the roundtrip loss to have an optimal extinction and is achieved with directional couplers with $180 \mathrm{~nm}$ gap. The AWG has 16 channels with $1.6 \mathrm{~nm}$ spacing, so that its bandwidth is larger than the envelope period of the sensor $(13 \mathrm{~nm})$ to avoid it from limiting the sensor's dynamic range. The chosen channel spacing is the result of a trade-off between having a high resolution and having enough spectral averaging per channel to be able to disclose the envelope signal directly with a broadband light source.

To couple light from and to the device, the input and output waveguides were adiabatically tapered to a $10 \mu \mathrm{m}$ wide ridge waveguide in which a second-order grating was etched to diffract TE-polarized light out-of-plane. The relative power diffracted to free space by all output gratings, each corresponding with a channel of the AWG, was monitored in parallel using a near-infrared camera with a microscope objective at a distance of several centimeters from the chip. A standard single-mode optical fiber was butt-coupled to the input grating.

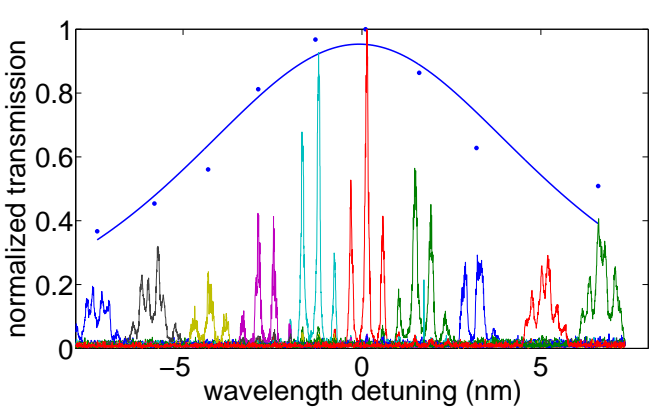

Fig. 2. The transmission spectrum of the Vernier-cascade through different channels of the AWG measured with a tunable laser (bottom, a color for each AWG-channel) shows the envelope peak superposed on the sharp constituent peaks. Approximately three constituent peaks are transmitted by each channel, providing enough power averaging per channel to also reveal the shape of the envelope peak when the transmission is measured with a broadband light source (blue dots). A squared Lorentzian function can be satisfactory fitted to the broadband transmission peak (blue line), allowing accurate determination of the peak position.

An envelope peak superposed on the constituent peaks is visible in the transmission spectrum of the watercovered Vernier-cascade sensor filtered by the AWG to its different channels (Fig. 2), confirming the on-chip spectral filtering and parallel camera read-out. The spectrum measured by stepping the wavelength of a tunable laser shows that approximately three constituent peaks are transmitted by each channel, providing enough power averaging to also reveal the envelope shape when the transmission spectrum is measured with a super-luminescent light emitting diode (LED) broadband light source with $1.55 \mu \mathrm{m}$ central wavelength. A squared Lorentzian function is satisfactory fitted to this envelope peak [9], allowing accurate determination of the peak position.

The performance of our device as transducer for labelfree biosensing was characterized by measuring the refractive index change of watery salt solutions in realtime. To allow controlled delivery of the solutions to the sensor, a microfluidic channel with $600 \mu \mathrm{m} \times 50 \mu \mathrm{m}$ cross section was made in polydimethylsiloxane (PDMS) by casting and directly bonded to the sensor chip. Using a syringe pump, the solutions were pumped at a $10 \mu \mathrm{L} / \mathrm{min}$ flow rate over both resonators of the Vernier-cascade to keep them both in thermal contact with the liquid. As the thermo-optic coefficient of the polymer cladding $\left(-1.5 \cdot 10^{-4} / K\right)$ is close to the thermo-optic coefficient of water $\left(-8 \cdot 10^{-5} / K\right)$, both resonators react similarly to changes in the liquid temperature. Hence our device is very tolerant to temperature changes and no thermal control was needed for the experiment. While the flow was repeatedly switched between deionized water and three different concentrations of $\mathrm{NaCl}$ in water, the broadband transmission of the Vernier-cascade sensor 
through the different channels of the AWG was measured with a suboptimal $6 \mathrm{~Hz}$ resolution and the spectral position of the transmission peak was determined by fitting the squared Lorentzian function mentioned before. The refractive index of the salt solutions was calculated according to ref. [12].

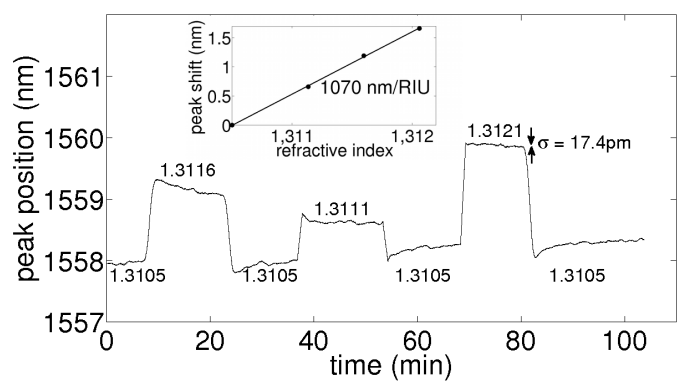

Fig. 3. The device can accurately monitor refractive index changes of watery $\mathrm{NaCl}$ solutions in real-time while being interrogated with a low-cost broadband light source, making it very applicable in low-cost labelfree biosensing. After smoothening the signal with a $1 \mathrm{~min}$ window, the standard deviation of the noise was only $17.4 \mathrm{pm}$. The sensor responded very linearly with $1070 \mathrm{~nm} / \mathrm{RIU}$ sensitivity (inset).

Our device allows to monitor refractive index changes fast and accurately (Fig. 3), despite the limited spectral resolution of the AWG and corresponding inaccuracy of the peak fitting. As the refractive index changes in label-free biosensing are typically slow compared to the period with which our spectra can be captured with a camera, the noise on the peak position signal can be reduced by smoothening the signal with a moving average over a time scale corresponding to the speed of the refractive index changes of interest. The central limit theorem in statistics predicts that the standard deviation on the signal will be reduced by the square root of the amount of spectra in the chosen window. We reduced the standard deviation of the noise on the signal to only $17.4 \mathrm{pm}$ by smoothening with a $1 \mathrm{~min}$ window. The spectral position of the transmission peak shifts very linearly with the refractive index of the solution with a sensitivity as high as $1070 \mathrm{~nm} / R I U$ (inset Fig. 3). This brings the detection limit of this device to $17.4 \mathrm{pm} / 1070 \mathrm{~nm} / \mathrm{RIU}=1.6 \cdot 10^{-5} \mathrm{RIU}$, which is in the same order as the detection limit we reported for a similar Vernier-sensor interrogated with a much more expensive tunable laser [9]. Moreover, it is expected to be an order of magnitude better than the interrogation with a broadband light source of the ring resonator sensor integrated with a photonic crystal spectral filter reported in [8], since our sensor is ten times more sensitive and can be resolved with a slightly better spectral resolution.

To conclude, the reported Vernier-cascade sensor with integrated AWG spectral filter achieves a detection limit that is low enough for many label-free biosensing appli- cations $\left(1.6 \cdot 10^{-5} R I U\right)$ while being interrogated with a low-cost broadband light source. The relaxation on the required complexity of the source moreover allows the integration of the source and detectors [6], further reducing the cost of the device and increasing its portability.

Future work will involve increasing the temporal resolution of the camera read-out, which was only $6 \mathrm{~Hz}$ in our experiment due to a software limitation. This is useful either to further improve the detection limit by reducing the noise on the sensor signal by averaging or to reduce the required length of the averaging window to allow monitoring fast molecular interactions. The drift of the sensor signal (Fig. 3), which was negative for salt solutions and positive for deionized water, also needs further investigation. Diffusion of ions in the polymer cladding is considered as a possible cause.

The authors thank ePIXfab (www.epixfab.eu) for the fabrication of the photonic chip, Steven Verstuyft for help during the final fabrication steps and Nebiyu Yebo for useful discussions. Tom Claes was supported by the Flemish Institute for the Promotion of Innovation through Science and Technology (IWT) with a specialization grant and Wim Bogaerts by the Flemish Research Foundation with a postdoctoral fellowship. This work was performed in the context of the European project InTopSens and the Belgian IAP project Photonics@BE.

\section{References}

1. A. J. Qavi, A. L. Washburn, J.-Y. Byeon, and R. C. Bailey, Anal. Bioanal. Chem. 394, 121 (2009).

2. K. De Vos, J. Girones, T. Claes, Y. De Koninck, S. Popelka, E. Schacht, R. Baets, and P. Bienstman, IEEE Photon. J. 1, 225 (2009).

3. M. Iqbal, M. A. Gleeson, B. Spaugh, F. Tybor, W. G. Gunn, M. Hochberg, T. Baehr-Jones, R. C. Bailey, and L. C. Gunn, IEEE J. Sel. Topics Quantum Electron. 16, 654 (2010).

4. D. X. Xu, M. Vachon, A. Densmore, R. Ma, A. Delage, S. Janz, J. Lapointe, Y. Li, G. Lopinski, D. Zhang, Q. Y. Liu, P. Cheben, and J. H. Schmid, Opt. Lett. 35, 2771 (2010).

5. B. Sepulveda, J. Sanchez del Rio, M. Moreno, F. J. Blanco, K. Mayora, C. Dominguez, and L. M. Lechuga, J. Opt. A: Pure Appl. Opt. 8, S561 (2006).

6. P. S. Petrou, M. Kitsara, E. Makarona, I. Raptis, S. E. Kakabakos, R. Stoffer, G. Jobst, and K. Misiakos, in Conf. Proc. IEEE Eng. Med. Biol. Soc. (2010), p. 298.

7. L. Jin, M. Li, and J.-J. He, Opt. Lett. 36, 1128 (2011).

8. Z. Xia, B. Momeni, M. Askari, M. Chamanzar, S. Yegnanarayanan, A. A. Eftekhar, and A. Adibi, in IEEE Photonics Annual Meeting (2010), pp. 329-330.

9. T. Claes, W. Bogaerts, and P. Bienstman, Opt. Express 18, 22747 (2010).

10. L. Jin, M. Li, and J.-J. He, Opt. Commun. 284, 156 (2011).

11. W. Bogaerts, S. K. Selvaraja, P. Dumon, J. Brouckaert, K. De Vos, D. Van Thourhout, and R. Baets, IEEE J. Sel. Topics Quantum Electron. 16, 33 (2010).

12. H. Su and X. G. Huang, Sens. Actuators, B 126, 579 (2007). 


\section{Informational Fourth Page}

\section{References}

1. A. J. Qavi, A. L. Washburn, J.-Y. Byeon, and R. C. Bailey, "Label-free technologies for quantitative multiparameter biological analysis", Anal. Bioanal. Chem., 394, 121 (2009).

2. K. De Vos, J. Girones, T. Claes, Y. De Koninck, S. Popelka, E. Schacht, R. Baets, and P. Bienstman, "Multiplexed Antibody Detection With an Array of Silicon-on-Insulator Microring Resonators", IEEE Photon. J. 1, 225 (2009).

3. M. Iqbal, M. A. Gleeson, B. Spaugh, F. Tybor, W. G. Gunn, M. Hochberg, T. Baehr-Jones, R. C. Bailey, and L. C. Gunn, "Label-Free Biosensor Arrays Based on Silicon Ring Resonators and High-Speed Optical Scanning Instrumentation", IEEE J. Sel. Topics Quantum Electron. 16, 654 (2010).

4. D. X. Xu, M. Vachon, A. Densmore, R. Ma, A. Delage, S. Janz, J. Lapointe, Y. Li, G. Lopinski, D. Zhang, Q. Y. Liu, P. Cheben, and J. H. Schmid, "Label-free biosensor array based on silicon-on-insulator ring resonators addressed using a WDM approach",Opt. Lett. 35, 2771 (2010).

5. B. Sepulveda, J. Sanchez del Rio, M. Moreno, F. J. Blanco, K. Mayora, C. Dominguez, and L. M. Lechuga, "Optical biosensor microsystems based on the integration of highly sensitive Mach-Zehnder interferometer devices", J. Opt. A: Pure Appl. Opt. 8, S561 (2006).

6. P. S. Petrou, M. Kitsara, E. Makarona, I. Raptis, S. E. Kakabakos, R. Stoffer, G. Jobst, and K. Misiakos, "Monolithically Integrated Biosensors based on Frequency-Resolved Mach-Zehnder Interferometers for Multi-analyte determinations", in Conf. Proc. IEEE Eng. Med. Biol. Soc. (2010), p. 298.

7. L. Jin, M. Li, and J.-J. He, "Optical waveguide doublering sensor using intensity interrogation with a low-cost broadband source", Opt. Lett. 36, 1128 (2011).

8. Z. Xia, B. Momeni, M. Askari, M. Chamanzar, S. Yegnanarayanan, A. A. Eftekhar, and A. Adibi, "Silicon Microring Resonator Sensor with Integrated PC Spectrometer for Sharp Spectral Features Detection", in IEEE Photonics Annual Meeting (2010), pp. 329-330.

9. T. Claes, W. Bogaerts, and P. Bienstman, "Experimental characterization of a silicon photonic biosensor consisting of two cascaded ring resonators based on the Vernier-effect and introduction of a curve fitting method for an improved detection limit", Opt. Express 18, 22747 (2010).

10. L. Jin, M. Li, and J.-J. He, "Highly-sensitive siliconon-insulator sensor based on two cascaded micro-ring resonators with vernier effect", Opt. Commun. 284, 156 (2011).

11. W. Bogaerts, S. K. Selvaraja, P. Dumon, J. Brouckaert, K. De Vos, D. Van Thourhout, and R. Baets, "Siliconon-Insulator Spectral Filters Fabricated With CMOS Technology", IEEE J. Sel. Topics Quantum Electron. 16, 33 (2010).

12. H. Su and X. G. Huang, "Fresnel-reflection-based fiber sensor for on-line measurement of solute concentration in solutions", Sens. Actuators, B 126, 579 (2007). 